\title{
DESIGN OPTIMIZATION OF THE HOLOS-QUAD MICRO-REACTOR CONCEPT
}

\author{
N. E. Stauff1*, C. H. Lee1, A. Wells2, C. Filippone2 \\ 1 Argonne National Laboratory \\ 9700 S. Cass Avenue Argonne, IL 60439, USA \\ 2 HolosGen LLC \\ Manassas Park, Virginia, USA \\ *nstauff@anl.gov
}

\begin{abstract}
The Holos-Quad micro-reactor concept is proposed by HolosGen LLC to generate $22 \mathrm{MWt}$ with a lifetime of approximately 8 effective full power years (EFPYs) for civilian applications. The design is based on a very innovative high-temperature gas-cooled reactor concept using four Subcritical Power Modules (SPMs) that fit into one commercial 40-foot transport ISO container. A rigorous design approach was employed in order to ensure that all input parameters were fully investigated and the best solutions possible were considered. The first step of this approach consisted of properly defining the Holos-Quad design problem by identifying the design objectives, the operational constraints, and the input parameters. In the second step, a sensitivity analysis was performed to enable a preliminary investigation of the input parameters to identify the correlations among input and output variables. Finally, the design optimization was performed in the third step, employing a genetic algorithm to effectively explore the highly constrained input parameters and find global optimal solutions. The multi-objective optimization identified various core solutions that would be optimum solutions for different types of applications. For applications where economics matters less and the ease of transportation matters more, a core weight of $\sim 15$ tons could achieve a lifetime of $\sim 3.5$ EFPYs. For applications where economics matters more and the ease of transportation matters less, a lifetime of 8.3 EFPYs could be achieved with a total core weight of $\sim 26.7$ tons.
\end{abstract}

KEYWORDS: micro-reactor, design optimization, HTGR

\section{INTRODUCTION}

Micro nuclear reactors are being developed for different applications such as deployment in harsh locations, long-term electricity supply to remote areas, emergency power in response to a natural disaster, etc. For rapid deployment to locations where the external power grid is weak or inexistent and access to operation personnel is limited, a very small reactor in terms of power rate and physical size is favorable. A power rate less than 20 MWe with all components manufactured, assembled and tested at a factory are preferred, altogether with a high level of passive safety and an autonomous reactor control system. In this context, HolosGen LLC is developing the Holos-Quad micro-reactor concept, a very innovative high-temperature gas-cooled reactor concept using four Subcritical Power Modules (SPMs) that fit into one commercial 40foot transport ISO container, to generate $22 \mathrm{MWt}$ with a lifetime of at least 8 effective full power years (EFPYs) for electricity production to support civilian applications. Under the ARPA-E MEITNER program initiated in FY2019, the Argonne National Laboratory (ANL) design team has been contributing to the core 
design of the Holos-Quad by performing a design optimization of the core using an innovative approach [1]. This multi-step procedure enables one to properly formulate the design problem and to ensure that the input space is fully investigated and that the best solutions are considered for any potential applications of the Holos-Quad concept. The steps of the design approach proposed consist of properly defining the design problem (step 1), sensitivity analysis (step 2) and multi-criteria analysis (step 3).

\section{DEFINITION OF THE CORE DESIGN PROBLEM}

The Holos-Quad is a high-temperature gas-cooled reactor (HTGR) concept generating $22 \mathrm{MWt}$ and designed to fully fit into one 40 -foot transport ISO container. The available width and height of the ISO container is limited to $2.34 \mathrm{~m}$, as shown in Figure 1. The length of the 40-foot ISO container $(12 \mathrm{~m})$ does not constrain the core design of the Holos-Quad. It uses TRISO fuel distributed in graphite hexagonal blocks and cooled with helium gas. The Holos-Quad reactor core under optimization in this paper is formed by four independent subcritical power modules (SPMs), enabling power generation when a minimum number of SPMs are actively positioned by actuators to satisfy criticality requirements, as illustrated in Figure 1. Each SPM contains its own closed-loop, gas Brayton cycle with a turbine, compressor, inter-cooler and rejection heat exchanger, generating 5.5 MW thermal power. In the configuration analyzed, each SPM is wrapped inside a shell of zircaloy-4 to prevent outside air from interacting with graphite at high temperature. Two reactivity control systems are being considered in this paper: one controls reactivity by moving the SPMs apart and increasing the neutron leakage, the other involves insertion of hafnium blades in between the SPMs as a complementary emergency shutdown system. Additional and complimentary control systems are being considered in the framework of the ARPA-E MEITNER program, but were not included in this preliminary optimization exercise.

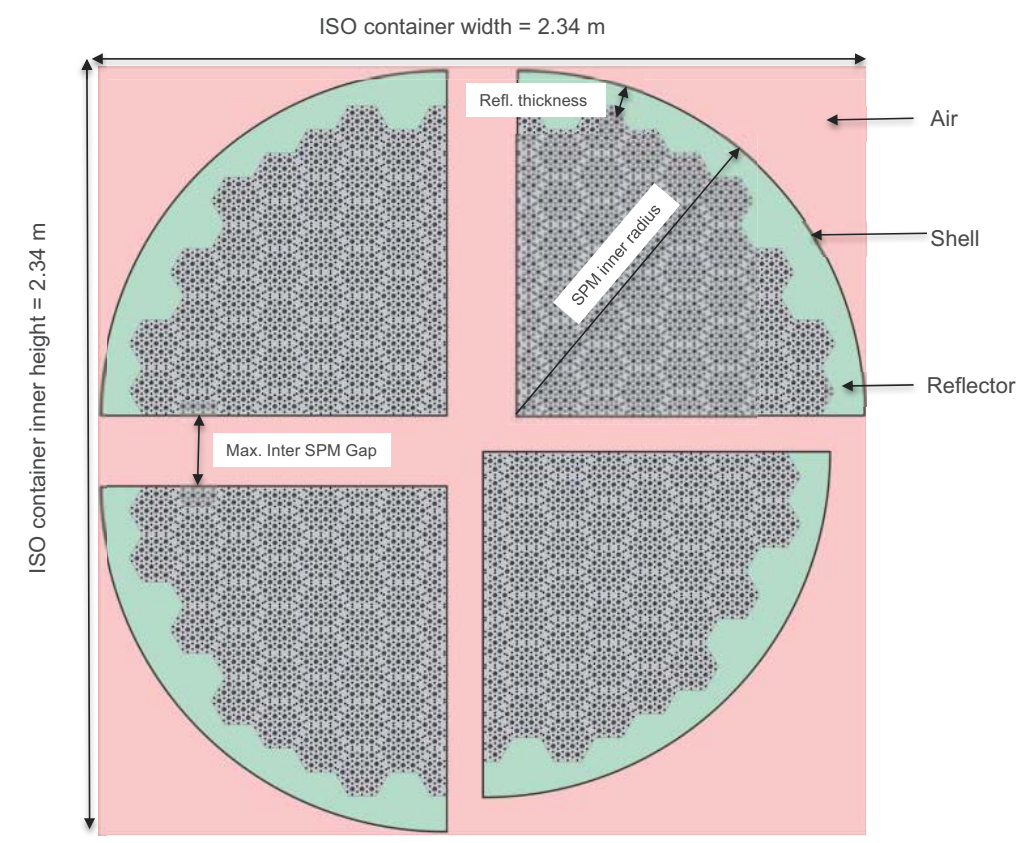

Figure 1. Transversal core layout with 3 out of 4 SPMs withdrawn for shutdown.

A few simplifications on the core model are considered at this stage of the core design. First, the graphite and beryllium blocks used in the fuel and reflector assemblies are assumed to be perfectly cut to fit into the cylindrical shell (see Figure 1). Second, no cooling material channel is currently considered in the radial reflector block. However, coolant channels are considered in the axial reflectors. Finally, the channels containing the flow of cold coolant returning to the compressor are not modelled. These channels will be 
positioned in a cavity between the radial reflector and the SPM outermost shell, alternatively being embedded with the radial reflector. For neutronics modeling consideration, three core configurations are considered with following changes in cross-section temperatures. At this stage of our study, changes in density (from thermal expansion) are not considered, and all dimensions provided in Table 1 are at hot temperature conditions.

- Full power: $\quad$ Fuel temp $=900 \mathrm{~K}$, Coolant + Structure (including $\mathrm{S}(\alpha, \beta))=800 \mathrm{~K}$

- Hot shutdown: Fuel temp $=800 \mathrm{~K}$, Coolant+Structure (including $\mathrm{S}(\alpha, \beta))=800 \mathrm{~K}$

- $\quad$ Cold shutdown: Fuel temp $=300 \mathrm{~K}$, Coolant+Structure (including $\mathrm{S}(\alpha, \beta)$ ) $=300 \mathrm{~K}$

The first step of a core design consists of properly defining the design problem, which means determining performance parameters to optimize the input variables and considering the design constraints. Those are detailed in this section and summarized in Figure 2.

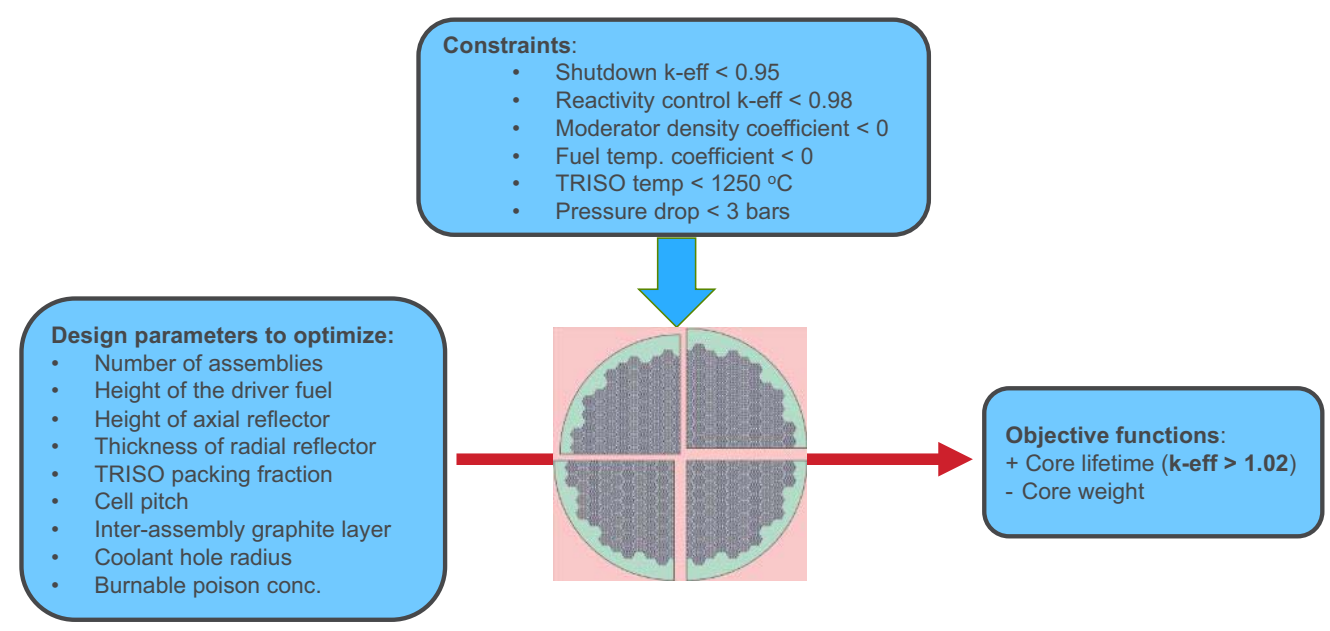

Figure 2. Description of the optimization problem.

\section{Optimization Goals}

The Holos-Quad parameters to be optimized are core life-time and core weight, as these competing metrics try to maximize two important aspects of the Holos-Quad concept: economic performance and transportability.

- Core Life-Time: The Holos-Quad is targeting long life-time in order to improve the economics of the system by enabling continuous operations for several years after installation. The core life-time is defined as the full power irradiation time over which the core maintains the effective multiplication factor (k-eff) larger than 1.02. The limit of 1.02 was decided to maintain operational flexibility even at End Of Life (EOL) and compensate for xenon poisoning. Consequently, this metric is a conservative value of the total irradiation life-time that would also need to consider increased life-time due to load following operation and potential life-time extension with reduced flexibility and reduced core power.

- Core Weight: The total core weight of the Holos-Quad should be limited in order to facilitate means of transportation to remote location, for example, by helicopter or airplane. The core weight is estimated as the total weight of material in the core, considering:

$\checkmark$ The driver fuel regions containing the weight of the TRISO particles, graphite, and coolant sleeves using zircaloy-4. 
The axial and radial reflector regions for which Beryllium metal was selected in this design confirguation for its low weight and excellent reflector performance, despite its lower melting temperature and toxicity.

$\checkmark$ The shell material structure, which is currently also made of zircaloy-4.

\section{Design Constraints}

The design constraints for the Holos-Quad concept are separated into 3 different sub-constraints:

- Reactivity shutdown: These simplified constraints consider the capability to shut down the core at any time of the irradiation. They account for potential increase in the k-eff value during irradiation due to the use of burnable poisons and for statistical uncertainty of the Monte Carlo solution. Two constraints are considered:

1. Cold shutdown (CSD): K-eff $<0.95$, considering maximum separation of the 4 SPMs with insertion of $1 \mathrm{~cm}$ thick hafnium blade using Eq. (1).

$$
\operatorname{Max}\left(k_{e f f}^{C S D}\right)=\operatorname{Max}\left(k_{e f f}(t)\right)-k_{e f f}(0)+k_{e f f}^{C S D}(0)+\frac{\sigma\left(k_{e f f}(0)\right)}{2}<0.95
$$

2. Hot shutdown (HSD): K-eff $<0.98$, considering maximum separation of only 3 out of 4 SPMs to model the impact of failure to move one SPM (Figure 1) using Eq. (2).

$$
\operatorname{Max}\left(k_{e f f}^{H S D}\right)=\operatorname{Max}\left(k_{e f f}(t)\right)-k_{e f f}(0)+k_{e f f}^{H S D}(0)+\frac{\sigma\left(k_{e f f}(0)\right)}{2}<0.98
$$

- Reactivity feedback constraints are evaluated at Beginning Of Life (BOL) where the MDC is more constraining due to the presence of burnable poisons diluted in the graphite moderator.

3. Moderator Density Coefficient $(\mathrm{MDC})<0$, calculated by reducing the graphite density of the fuel pin matrix and the graphite block by $1 \%$ (density of Be reflector is not changed) and accounting for statistical uncertainty with Eq. (3).

$$
\frac{1}{k_{e f f}^{R e f}(0)}-\frac{1}{k_{e f f}^{M D C}(0)}+\frac{\sqrt{\sigma\left(k_{e f f}^{R e f}(0)\right)^{2}+\sigma\left(k_{e f f}^{M D C}(0)\right)^{2}}}{2}<0
$$

4. Fuel Temperature Coefficient $(\mathrm{FTC})<0$, calculated by increasing the temperature of the UCO fuel by $300 \mathrm{~K}$ (from $900 \mathrm{~K}$ to $1200 \mathrm{~K}$ ) and accounting for statistical uncertainty with Eq. (4).

$$
\frac{1}{k_{e f f}^{R e f}(0)}-\frac{1}{k_{e f f}^{F T C}(0)}+\frac{\sqrt{\sigma\left(k_{e f f}^{R e f}(0)\right)^{2}+\sigma\left(k_{e f f}^{F T C}(0)\right)^{2}}}{2}<0
$$

- Thermal hydraulics constraints are estimated on the hot channel, considering radial power peaking of 1.5868 , and axial power peaking of 1.1821. Both power peaking values were evaluated with PROTEUS $[2,3]$ on an early version of the Holos-Quad design and should be relatively realistic values for the first round of design optimization study.

5. Peak Pressure Drop $<3$ bars

6. Nominal Peak TRISO Temperature $<1250$ oC

- Flooding constraint: An additional reactivity shutdown constraint that needs to be considered is the capability to remain sub-critical in cold shutdown state during flooding events (water ingression scenarios) around the SPMs and inside the coolant channels. Water ingression brings additional reactivity in under-moderated cores (since graphite is not as good a moderator as water). This scenario is evaluated but not currently used as a hard constraint in this optimization problem since doing so requires design of secondary control system that will bring additional shutdown margins. 


\section{Input Parameters}

The design parameters that were fixed or varied are listed in Table 1. For the varied parameters, the upper and lower bounds and the number of points within this range used in the optimization are listed as well. As discussed in Reference [1], the convergence of the optimization calculation is improved if performed on discrete variables instead of continuous ones.

Table 1. Design parameters used for modeling the Holos-Quad core (hot conditions).

\begin{tabular}{|c|c|c|c|c|c|}
\hline & Parameters & Unit & $\begin{array}{c}\text { Value / Variable } \\
\text { name }\end{array}$ & $\begin{array}{c}\text { Lower/Upper } \\
\text { Bound }\end{array}$ & $\begin{array}{l}\text { Number } \\
\text { of Points }\end{array}$ \\
\hline \multirow{2}{*}{$\begin{array}{l}\text { Shell } \\
\text { (Zirc-4) }\end{array}$} & Thickness & $\mathrm{cm}$ & 0.5 & & \\
\hline & Inner radius & $\mathrm{m}$ & evaluated & & \\
\hline \multirow{5}{*}{ Core } & Power & MWt & 22 & & \\
\hline & $\begin{array}{l}\text { Number of full/half/quarter fuel } \\
\text { columns }\end{array}$ & & varied (NA) & $151 / 249$ & 10 \\
\hline & Height of the fuel column & $\mathrm{m}$ & $\operatorname{varied}(\mathrm{H})$ & $200 / 500$ & 31 \\
\hline & Height of upper/lower reflector & $\mathrm{m}$ & varied $(h)$ & $5 / 25$ & 5 \\
\hline & $\begin{array}{l}\text { Estimated thickness of radial } \\
\text { reflector }\end{array}$ & $\mathrm{cm}$ & varied $(T r)$ & $\begin{array}{c}5 / 15 \text { (constrained } \\
\text { by geom.) }\end{array}$ & 11 \\
\hline \multirow{4}{*}{$\begin{array}{l}\text { Fuel } \\
\text { block }\end{array}$} & Pitch & $\mathrm{cm}$ & evaluated & & \\
\hline & Number of fuel holes & & 19 & & \\
\hline & Number of small coolant holes & & 54 & & \\
\hline & Inter-assembly graphite layer & $\mathrm{cm}$ & varied (It) & $0.05 / 0.40$ & 8 \\
\hline \multirow{7}{*}{ Fuel cell } & Pitch of fuel cell & $\mathrm{cm}$ & varied $(p)$ & $1.4 / 1.75$ & 8 \\
\hline & Radius of fuel hole & $\mathrm{cm}$ & 0.7 & & \\
\hline & Packing fraction & $\%$ & varied & $20 / 40$ & 2 \\
\hline & Fuel form & & $\begin{array}{c}\mathrm{UCO}, \mathrm{C} / \mathrm{U}=0.4 \\
\mathrm{O} / \mathrm{U}=1.5\end{array}$ & & \\
\hline & Outside radius of coolant hole & $\mathrm{cm}$ & varied $(r c)$ & $0.30 / 0.38$ & 6 \\
\hline & Coolant cladding (Zirc-4) thickness & $\mathrm{cm}$ & 0.057 & & \\
\hline & $\begin{array}{l}\text { Lead buffer thickness (between } \\
\text { coolant cladding and graphite) }\end{array}$ & $\mu \mathrm{m}$ & 20 & & \\
\hline \multirow{5}{*}{$\begin{array}{l}\text { Particle } \\
\text { size }\end{array}$} & Fuel kernel diameter (UCO) & \multirow{5}{*}{$\mu \mathrm{m}$} & 425 & & \\
\hline & Buffer thickness & & 100 & & \\
\hline & Inner pyrolytic carbon thickness & & 40 & & \\
\hline & Silicon carbide thickness & & 35 & & \\
\hline & Outer pyrolytic carbon thickness & & 40 & & \\
\hline \multirow{11}{*}{$\begin{array}{l}\text { Material } \\
\text { density }\end{array}$} & Fuel kernel (UCO) & \multirow{11}{*}{$\mathrm{g} / \mathrm{cm}_{3}$} & 10.744 & & \\
\hline & Buffer & & 1.04 & & \\
\hline & Inner pyrolytic carbon & & 1.882 & & \\
\hline & Silicon carbide $(\mathrm{SiC})$ & & 3.171 & & \\
\hline & Outer pyrolytic carbon & & 1.882 & & \\
\hline & Graphite block (matrix) & & 1.806 & & \\
\hline & Reflector block (Beryllium) & & 1.778 & & \\
\hline & Coolant $\mathrm{He}$ & & 0.00365 & & \\
\hline & Air (inter-modules $-\mathrm{N}_{2}$ ) & & 0.0012 & & \\
\hline & Lead & & 10.253 & & \\
\hline & Zircaloy-4 (SPM shell) & & 6.489 & & \\
\hline \multicolumn{2}{|c|}{ B-10 burnable absorbent diluted in graphite } & ppm & varied $(B P)$ & $10 / 15$ & 13 \\
\hline
\end{tabular}




\section{METHODS AND CODES DESCRIPTION}

\subsection{Calculation Codes and Methods}

The Monte Carlo code SERPENT [4] is the main code employed for neutronic simulations for core design and analysis in this work. It is a continuous-energy Monte-Carlo reactor physics burnup calculation code developed at the VTT Technical Research Center, Finland. In this analysis, SERPENT is used for detailed core modeling, employing the ENDF/B-VII.1 nuclear data library. The fuel region is modeled with an explicit description of the TRISO fuel where particles are distributed with semi-random sampling technique; they are filled in a compact regular lattice, and random particles are then removed to meet the packing fraction requested. For the burnup depletion calculation, the full core is depleted using a unique depletion region in the core optimization and neutronic benchmark analysis. Verification of the calculation methods was accomplished by performing neutronic benchmarks against the stochastic (OpenMC) and deterministic (PROTEUS) codes on the optimized core configuration $[3,8]$.

The System Analysis Module (SAM) [5] is a modern system analysis tool under development at ANL for advanced non-LWR safety analysis. The SAM PBCoreChannel component was used to model both coolant channel (1D) and heat structures (2D). The HolosGen fuel assembly is modeled by a representative pincell, which represents an average fuel pin, graphite, buffer, cladding, and coolant channel. In the SAM model, a homogenized fuel region was assumed, although the fuel was compacted with the TRISO particles. To obtain the kernel temperature of TRISO particles, the analytical temperature distribution of the spherical geometry was applied using the SAM-obtained fuel temperature as an external boundary condition.

\subsection{Optimization Framework Description}

Solving the core design optimization problem above described can be manually executed through a sensitivity analysis, which requires good understanding of the physics and manual testing of many design configurations. However, for systematic investigation of the input space, we employed a formal mathematical optimization approach that had been recently developed [1] and applied to sodium-cooled fast reactor (SFR) core design. The full input space considered with the parameters listed in Table 2 contains 150 million options. An optimization algorithm is able to navigate this input space efficiently to converge to the best options within limited runs. Still, the main challenge of such approach is to be relatively computationally expensive since $\sim 2,500$ cores will be modeled in Section 5 to reach acceptable convergence. However, the main benefit of this approach is to significantly reduce human effort (after the initial setup of the problem) and to be able to automatically re-run the optimization when changing the design problem (with different performance targets, new input parameters, relaxed constraints, etc.).

The PyHolos software introduced in Figure 3 was developed using Python to take design parameters and to generate, execute and post-process simultaneous SAM [5] and SERPENT [4] calculations in order to evaluate all the parameters of interest discussed in Section 2 (core lifetime, weight, reactivity coefficients, etc.). The PyHolos code can be adapted with little effort to any changes in the Holos core design, for instance, to change the number of SPMs, location of the hafnium blade, changes in reactivity control strategy, etc. The main benefit of PyHolos is to enable coupling with the DAKOTA code [6], as shown in Figure 3. The DAKOTA software maintained by Sandia National Laboratory is an uncertainty quantification and optimization toolkit that provides advanced mathematical methods to vary one code's input parameters and analyze the output results, enabling multi-objective optimization and uncertainty quantification analysis. DAKOTA samples each input parameters with the Latin Hypercube Sampling (LHS) technique [7] and generates the user-defined number of PyHolos inputs, which formulates the initial population. The driver interface executes the PyHolos code with the generated inputs and extracts output responses of interest through individual runs of SAM and SERPENT. Multiple inputs can be executed at the same time at this step. Once the calculations of all inputs are finished, DAKOTA analyzes the extracted 
output responses. For core optimization analysis, DAKOTA employs a genetic algorithm to generate the inputs of the next generation. This iterative procedure continues until optimization convergence is achieved, with the final generation being the sets of optimal solutions.

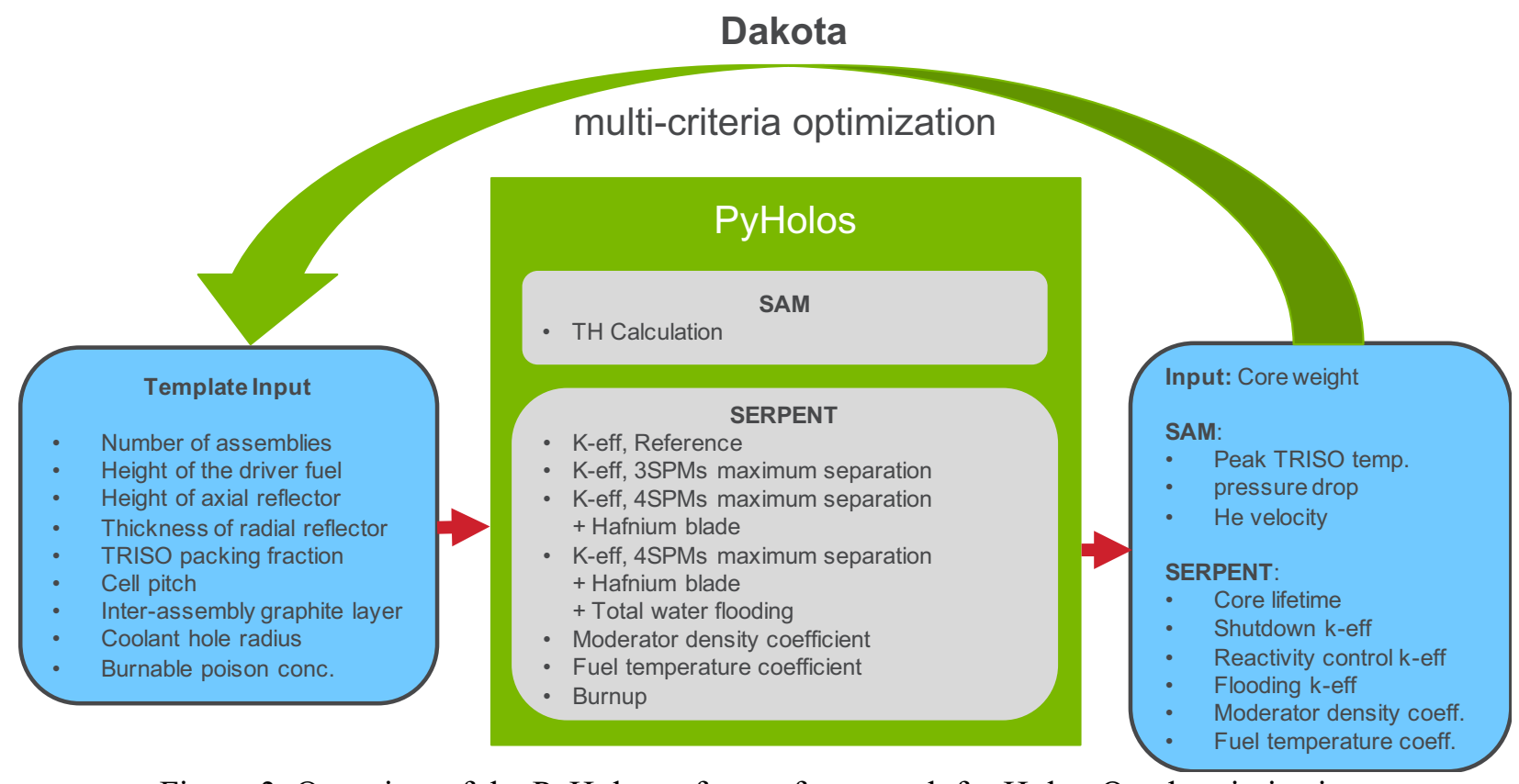

Figure 3. Overview of the PyHolos software framework for Holos-Quad optimization.

\section{SENSITIVITY STUDY}

Prior to running the full optimization problem (performed in Step 3), a global sensitivity analysis is suggested as the second step to investigate the correlations among core output variables, which represents the global trade-off among core optimization objectives. The sensitivity coefficients between inputs and outputs, which represent the impact of variation in input on the variation of output, is also calculated in this step to identify the important core design parameters of the optimization problem. The Latin Hypercube Sampling (LHS) technique implemented in DAKOTA was used to perform the sensitivity analysis. The results from the sensitivity analysis shown in Table 2 indicate that out of 1,000 simulated cores, only 117 meet all constraints, displaying a core lifetime larger than 0 . All of the designs considered meet the thermal hydraulics constraints. However, $24 \%$ did not meet the reactivity feedback constraints due to a positive MDC. Among those meeting the reactivity feedback constraints, only $30 \%$ met the reactivity shutdown constraints, mainly due to the inability to compensate the excess reactivity with sufficient margins at hot shutdown.

Table 2. Number of cores sampled meeting design constraints.

\begin{tabular}{|l|c|c|}
\hline $\begin{array}{l}\text { Number of samples } \\
\text { (LHS sampling) }\end{array}$ & All results & lifetime $>\boldsymbol{0}$ \\
\hline Meet thermal-hydraulics constraints & 1000 & $\mathbf{7 2 1}$ \\
Meet reactivity feedback constraints & 765 & 721 \\
Meet reactivity shutdown constraints & 224 & 589 \\
\hline
\end{tabular}

Finally, the results from the sensitivity analysis can be used to compute the Pearson correlation matrix within the different inputs and outputs of each simulation, as shown in Table 3. As expected, there is strong negative correlation between the number and length of the fuel cartridge, and the pressure drop and peak 
temperature in the TRISO fuel (i.e., more and longer coolant channels lead to reduced power density and flow rate, and reduced temperature and pressure drop). Such sensitivity analysis is especially useful to determine that parameters such as the axial reflector length and the inter-assembly graphite layer do not impact significantly the performance of the core and could be removed from the optimization problem to simplify it and to accelerate its convergence.

Table 3. Pearson correlations between Inputs and Outputs.

\begin{tabular}{|c|c|c|c|c|c|c|c|c|c|c|c|c|c|c|c|c|c|c|c|}
\hline & & \multicolumn{9}{|c|}{ Input Parameters } & \multicolumn{2}{|c|}{ pptimization Goals } & \multicolumn{7}{|c|}{ Design Constraints } \\
\hline & & NA & H & $\mathrm{h}$ & It & $\mathrm{p}$ & rc & $\mathrm{BP}$ & PF & $\operatorname{Tr}$ & Weight & Lifetime & Control & MDC & FTC & Shutdown & Flooding & \begin{tabular}{|c|} 
Pressure \\
Drop \\
\end{tabular} & $\begin{array}{l}\text { TRISO } \\
\text { Temp }\end{array}$ \\
\hline \multirow{2}{*}{$\begin{array}{c}\text { Optimization } \\
\text { Goal }\end{array}$} & Weight & 0.2 & 0.9 & 0.2 & 0.0 & 0.2 & 0.0 & 0.0 & 0.0 & 0.1 & 1.0 & & & & & & & & \\
\hline & Lifetime & 0.0 & 0.4 & 0.1 & 0.0 & 0.1 & -0.1 & -0.5 & 0.4 & 0.1 & 0.5 & 1.0 & & & & & & & \\
\hline \multirow{7}{*}{$\begin{array}{l}\text { Design } \\
\text { Constraints }\end{array}$} & \begin{tabular}{|l|} 
Control Rod \\
\end{tabular} & 0.3 & 0.2 & 0.1 & 0.1 & 0.4 & -0.1 & -0.4 & 0.2 & 0.3 & 0.4 & 0.6 & 1.0 & & & & & & \\
\hline & $\mathrm{MDC}$ & -0.1 & 0.1 & 0.1 & 0.0 & 0.0 & -0.1 & 0.3 & -0.3 & 0.2 & 0.1 & -0.2 & 0.0 & 1.0 & & & & & \\
\hline & FTC & -0.1 & 0.0 & 0.0 & 0.0 & 0.6 & -0.1 & -0.1 & -0.6 & 0.1 & 0.1 & -0.1 & 0.2 & 0.4 & 1.0 & & & & \\
\hline & Shutdown & 0.0 & 0.2 & 0.1 & 0.0 & 0.1 & -0.3 & -0.3 & 0.4 & 0.2 & 0.3 & 0.7 & 0.8 & 0.0 & 0.0 & 1.0 & & & \\
\hline & Flooding & 0.1 & 0.1 & 0.0 & 0.0 & -0.4 & 0.1 & -0.5 & 0.7 & 0.0 & 0.1 & 0.6 & 0.4 & -0.3 & -0.5 & 0.7 & 1.0 & & \\
\hline & Pressure Dro: & -0.4 & 0.4 & 0.0 & 0.0 & 0.0 & -0.7 & 0.0 & 0.0 & 0.0 & 0.3 & 0.3 & 0.0 & 0.2 & 0.1 & 0.3 & -0.1 & 1.0 & \\
\hline & TRISO temp & -0.4 & -0.9 & 0.0 & 0.0 & 0.0 & 0.1 & 0.0 & -0.1 & 0.0 & -0.9 & -0.5 & -0.3 & 0.0 & 0.1 & -0.3 & -0.2 & -0.3 & 1.0 \\
\hline
\end{tabular}

\section{MULTI-CRITERIA CORE DESIGN OPTIMIZATION}

The main objective of the third step is to determine the best core options with minimum core weight at different targeted lifetimes while meeting all the operational constraints. To solve this multi-criteria optimization problem, a genetic algorithm was applied due to its capability to effectively explore a highly constrained input space to find global optimal solutions. The results in Figure 4 show the evolution of the performance of the best set of 200 cores for each generation ( 35 generations were considered). This figure illustrates that each point represents a unique fully characterized core and that long core life-time is achievable with larger core weight, confirming that these objective functions are competing against each other. The calculation was stopped at generation 35 (requiring a total of $>2500$ core simulations) when the performance improvements of the cores in the Pareto Front (clustering of the optimum solutions) was found to be limited. Core designs located in Pareto Frontier are optimal solutions suggested by the multi-objective optimization method, each of which reflects the trade-off among the two performance criteria considered in this design problem. Using current technologies and sets of constraints, for example, the core lifetime of $\sim 3.5$ EFPYs can be achieved with a total core weight of $\sim 15$ tons. If the core weight is increased to 30 tons, the lifetime achievable is $\sim 9$ EFPYs.

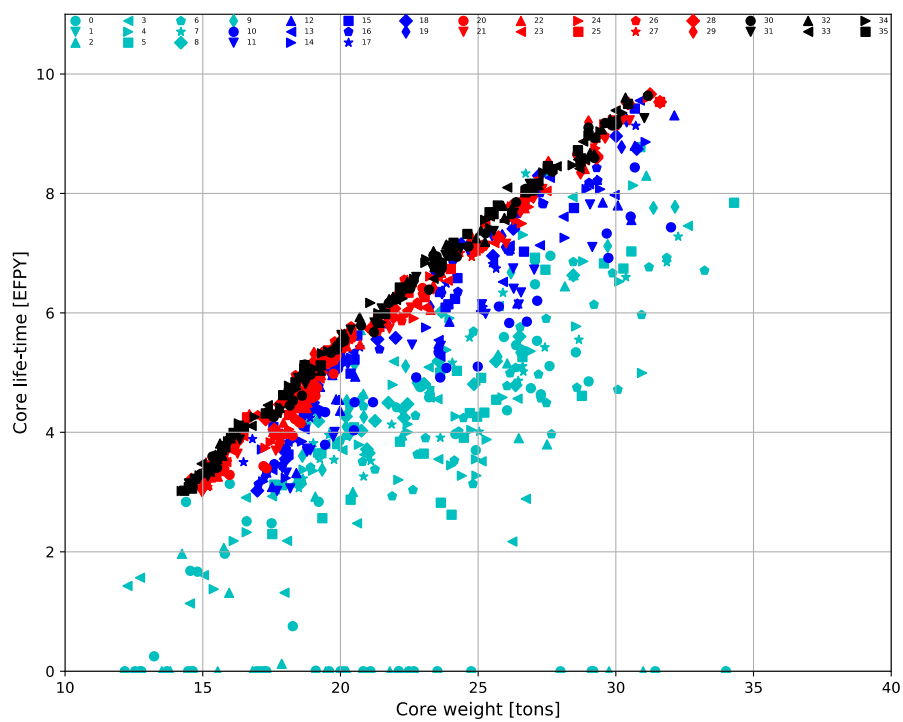

Figure 4. Pareto Frontier obtained from the multi-criteria optimization. Each generation of solution is shown with different shapes and color. 
A few promising input solutions are displayed in Table 4 with increasing life-time achieved. As observed in this table, all the core concepts meet the design constraints. It is interesting to note that all the design options of the Pareto Front converged to the same number of fuel assembly (151: minimum number provided in the optimization problem), of packing fraction (0.4: maximum number provided in the optimization problem), and of coolant hole radius $(0.3 \mathrm{~cm}$ : minimum number provided in the optimization problem). Consequently, achieving better performance would be possible by extending the input space with reduced number of fuel assemblies and reduced coolant radius. However, such reductions will further increase the pressure drop, which will reduce the thermal efficiency of the Brayton cycle. This economic tradeoff was not considered at this time but should be accounted for in the future. To meet the lifetime objective of 8 years defined under the ARPA-E MEITNER program for the Holos-Quad, the core concept "Opt 8 " with a total weight of $\sim 26.7$ tons was selected for neutronic benchmark [3].

Table 4. Sample of optimum core solutions.

\begin{tabular}{|l|c|c|c|c|c|c|c|c|}
\hline & Opt 3 & Opt 4 & Opt 5 & Opt 6 & Opt 7 & Opt 8 & Opt 9 \\
\hline \multicolumn{7}{|c|}{ Input variables } \\
\hline Height of driver fuel [cm] & 210 & 230 & 270 & 330 & 360 & $\mathbf{3 9 0}$ & 440 \\
\hline Height of Axial reflectors [cm] & 5 & 5 & 5 & 5 & 5 & $\mathbf{5}$ & 5 \\
\hline Inter-assembly graphite layer [cm] & 0.25 & 0.2 & 0.2 & 0.4 & 0.25 & $\mathbf{0 . 2 5}$ & 0.25 \\
\hline Cell pitch [cm] & 1.65 & 1.7 & 1.7 & 1.65 & 1.7 & $\mathbf{1 . 7}$ & 1.7 \\
\hline External coolant hole radius [cm] & 0.3 & 0.3 & 0.3 & 0.3 & 0.3 & $\mathbf{0 . 3}$ & 0.3 \\
\hline Burnable poison concentration [ppm] & 10 & 13 & 16 & 16 & 10 & $\mathbf{2 0}$ & 11 \\
\hline TRISO packing fraction & 0.4 & 0.4 & 0.4 & 0.4 & 0.4 & $\mathbf{0 . 4}$ & 0.4 \\
\hline Thickness of radial reflector [cm] & 8 & 8 & 7 & 6 & 5 & 7 & 5 \\
\hline Number of Assemblies & 151 & 151 & 151 & 151 & 151 & $\mathbf{1 5 1}$ & 151 \\
\hline \multicolumn{7}{|c|}{ Performance } \\
\hline Core Weight [tons] & 14.2 & 16.2 & 18.6 & 21.6 & 23.8 & $\mathbf{2 6 . 7}$ & 29.0 \\
\hline Lifetime [EFPY] & 3.0 & 4.0 & 5.0 & 6.0 & 7.1 & $\mathbf{8 . 3}$ & 9.2 \\
\hline \multicolumn{7}{|c|}{ Constraints } \\
\hline 1- Control K-eff (CSD) & 0.92 & 0.95 & 0.94 & 0.91 & 0.96 & $\mathbf{0 . 9 7}$ & 0.97 \\
\hline 2- Shutdown K-eff (HSD) & 0.83 & 0.84 & 0.83 & 0.81 & 0.84 & $\mathbf{0 . 8 5}$ & 0.85 \\
\hline 3- MDC [pcm/\%] & -234 & -174 & -188 & -112 & -167 & $\mathbf{- 1 2 0}$ & -105 \\
\hline 4- FTC [pcm/300K] & -934 & -862 & -912 & -848 & -782 & $\mathbf{- 9 3 4}$ & -752 \\
\hline 5- Peak pressure drop [bar] & 0.36 & 0.39 & 0.46 & 0.56 & 0.61 & $\mathbf{0 . 6 5}$ & 0.74 \\
\hline 6- Peak TRISO temp [Celsius] & 989 & 967 & 935 & 902 & 889 & $\mathbf{8 7 9}$ & 865 \\
\hline
\end{tabular}

\section{CONCLUSIONS}

The Holos-Quad micro-reactor concept is based on a very innovative 22 MWt HTGR design using four SPMs that fit into one commercial 40 -foot ISO transport container. A rigorous design approach was employed at ANL in order to ensure that the very highly constrained input space is thoroughly investigated to identify the best possible solutions. The first step of this approach consisted of properly defining the Holos-Quad design problem by identifying the competitive design objectives: its economic performance was maximized with longer lifetime, and its transportability to remote locations was maximized with reduced weight. The design problem also required identifying the operational constraints (in terms of thermal hydraulics, reactivity feedback and shutdown margin) and the input parameters that could be varied. In the second step, a sensitivity analysis was performed to enable a preliminary investigation of the input parameters to identify the correlations among input and output variables. This allowed, for instance, to find input parameters that have relatively small impact on the core performance and that could be removed from 
the optimization problem to accelerate its convergence. This sensitivity analysis showed that the design problem defined was highly constrained since only 117 out of 1,000 simulated cores met all the design constraints.

The design optimization was performed in the third step, employing a genetic algorithm to effectively explore the highly constrained input parameters and find global optimal solutions. More than 2,500 cores were fully evaluated, which converged to a Pareto Frontier of optimum solutions providing the best compromise between the life-time target and the total core weight. The main benefit of this approach is to significantly reduce the labor-intense efforts normally required to execute these analyses and to be able to automatically re-run the optimization problem when changing the design problem. The multi-objective optimization identified various core solutions that would be optimum solutions for different types of applications. For applications where economics matters less and the ease of transportation matters more, a core weight of $\sim 15$ tons could achieve a lifetime of $\sim 3.5$ EFPYs. For applications where economics matters more and the ease of transportation matters less, a lifetime of 8.3 EFPYs could be achieved with a total core weight of $\sim 26.7$ tons. This latter design was determined to be the best configuration that would meet the design requirements of the ARPA-E MEITNER program and this configuration is being analyzed in further detail. Future work will include evaluation of additional and complimentary reactivity control mechanisms, various modifications in the core parameters, and will consider re-running this optimization procedure on a more detailed core model.

\section{ACKNOWLEDGMENTS}

Argonne National Laboratory's work was supported by U.S. Department of Energy (DOE) under Contract number DE-AC02-06CH11357. The direction and support of the U.S. DOE Office of Nuclear Energy and the ARPA-E MEITNER Program is greatly appreciated.

\section{REFERENCES}

1. Kaiyue Zeng et al., "Development of multi-objective core optimization framework and application to sodium-cooled fast test reactors," Progress in Nuclear Energy, Vol 120, February (2020) 103184.

2. Y. S. Jung et al., "PROTEUS-MOC User Manual (Rev.0)," ANL/NSE-18/10, Argonne National Laboratory (2018).

3. Nicolas E. Stauff et al., "Neutronic Benchmark on Holos-Quad Micro-Reactor Concept," PHYSOR 2020, Cambridge, United Kingdom, March 29-April 2 (2020).

4. J. Leppanen, "Serpent - a Continuous-energy Monte Carlo Reactor Physics Burnup Calculation Code," User's Manual, June 18 (2015).

5. R. Hu, "An advanced one-dimensional finite element model for incompressible thermally expandable flow," Nuclear Technology, Vol. 190, June (2015).B. Adams et al., "Dakota, A Multilevel Parallel Object-Oriented Framework for Design Optimization, Parameter Estimation, Uncertainty Quantification, and Sensitivity Analysis: Version 6.5 User's Manual," Sandia National Laboratory, 2016.

6. J. C. Helton, and F.J. Davis, "Latin hypercube sampling and the propagation of uncertainty in analyses of complex systems." Reliability Engineering and System Safety, Vol. 81, pp. 23-69 (2003).

7. N. Stauff et al., "Neutronic Design and Analysis of the Holos-Quad Concept," ANL/NSE-19/8, Argonne National Laboratory (2019). (https://www.osti.gov/biblio/1524786-neutronic-designanalysis-holos-quad-concept) 